\title{
Patient-reported outcomes of femoral osteotomy and total hip arthroplasty for osteonecrosis of the femoral head: a prospective case series study
}

\author{
Yusuke Kubo ${ }^{1}$, Takuaki Yamamoto ${ }^{2 *} \mathbb{D}$, Goro Motomura' ${ }^{1}$ Kazuyuki Karasuyama', Kazuhiko Sonoda' \\ and Yukihide Iwamoto ${ }^{3}$
}

\begin{abstract}
Background: Patient-reported scoring systems have recently been used after surgical procedures. The purpose of this prospective study was to evaluate the patient-reported outcomes of femoral osteotomy and total hip arthroplasty (THA) for osteonecrosis of the femoral head (ONFH).

Methods: Forty-two symptomatic ONFH patients with asymptomatic contralateral hip underwent either transtrochanteric anterior rotational osteotomy (ARO) or THA as a primary operation. Of these, 20 patients whose contralateral hips remained asymptomatic at the final follow-up (more than 1 year postoperatively) were recruited to participate in this study. Nine patients were treated with ARO (ARO group) and 11 patients were treated with THA (THA group). Both the Oxford hip score (OHS) and the short form 36 (SF-36) were evaluated preoperatively and at the final follow-up.

Results: The preoperative OHS was $29.1 \pm 10.9$ and $21.9 \pm 9.6$ points in the ARO and THA groups, which significantly improved to $38.4 \pm 9.4$ and $40.3 \pm 5.1$ points at the final follow-up, respectively. The preoperative physical component summary score was $30.8 \pm 12.8$ and $17.8 \pm 14.5$ points in the ARO group and THA groups, which significantly improved to $44.5 \pm 10.6$ and $43.3 \pm 10.4$ points at the final follow-up, respectively. The preoperative mental component summary score was $48.0 \pm 8.5$ and $48.6 \pm 11.3$ points in the ARO and THA groups, both of which remained unchanged at the final follow-up.
\end{abstract}

Conclusions: The short-term patient-reported outcomes of this study suggested that both ARO and THA for ONFH resulted in significantly improved postoperative hip joint function.

Keywords: Patient-reported outcomes, Osteotomy, Total hip arthroplasty, Osteonecrosis of the femoral head, Oxford hip score, Short form 36

\section{Background}

Osteonecrosis of the femoral head (ONFH) often leads to collapse of the femoral head, which usually causes severe hip pain and thus requires some kind of surgical treatment (Mankin 1992; Mont et al. 1995; Fukushima et al. 2010). Surgeries for collapsed ONFH are classified in two

\footnotetext{
*Correspondence: yamataku@ortho.med.kyushu-u.ac.jp

${ }^{2}$ Department of Orthopaedic Surgery, Faculty of Medicine, Fukuoka

University, 7-45-1 Nanakuma, Jonan-ku, Fukuoka, Japan

Full list of author information is available at the end of the article
}

major categories: total hip arthroplasty (THA) and jointpreserving surgeries (Sugioka 1978; Mont et al. 1995). The worldwide standard surgical procedure for ONFH is THA. This procedure has a wide application for collapsed ONFH, especially in cases at advanced stages of ONFH (Wiklund and Romanus 1991; Mont et al. 1995). On the other hand, in the early postcollapse stages of ONFH, hip joint preservation remains a preferred treatment option.

Transtrochanteric anterior rotational osteotomy (ARO), developed by Sugioka in 1972, is one of the joint-preserving alternatives for ONFH (Sugioka 1978). In patients 
with necrotic lesions of the anterosuperior aspect of the femoral head, the anterior rotation of the femoral head enables relocation of the weight-bearing region of the hip joint to the intact posterior aspect of femoral head (Sugioka 1978). To date, many studies have focused on ARO with the objective of improving the performance of surgery, which translates into an improvement of the joint preservation rate (Inao et al. 1999; Mont et al. 2006). In these studies, physician-reported scoring systems were commonly used as the surgical assessment.

To the best of our knowledge, no previously published studies have prospectively examined the perioperative patient-reported outcomes of symptomatic ONFH in patients with an asymptomatic contralateral hip who underwent either ARO or THA as the primary operation. These evaluations are considered to be useful for patients at the early postcollapse stages, particular in the decision-making process of whether to preserve the joint or not. In this study, we prospectively evaluated the patient-reported outcomes of ARO and THA for ONFH.

\section{Methods \\ Patients}

Forty-two symptomatic ONFH patients with asymptomatic contralateral hip were prospectively assessed for perioperative patient-reported outcomes. They underwent either ARO or THA as the primary operation for ONFH at our institution between June 2009 and June 2014. Of these, 20 patients whose contralateral hip remained asymptomatic at the final follow-up (which was variable, but lasted more than 1 year postoperatively) were recruited to participate in this study. They consisted of nine patients treated with ARO (ARO group) and 11 patients treated with THA (THA group) (Table 1). In the ARO group, there were eight men and one woman with a mean age of 38 years (range: $28-45$ ) at the time of surgery and a mean body mass index (BMI) of $24.1 \mathrm{~kg} / \mathrm{m}^{2}$. In the THA group, there two men and nine women with a mean age of 45 years (range: $29-60$ ) at the time of surgery and a mean BMI of $21.8 \mathrm{~kg} / \mathrm{m}^{2}$. The mean preoperative period, defined as the time from the onset of the hip pain until surgery, was 4.8 and 9.3 months in the ARO and THA groups, respectively. The mean postoperative follow-up period was 2.5 years (range: $1-6$ ) and 1.7 years (range: 1-4) in the ARO and THA groups, respectively.

The diagnosis of ONFH was based on the diagnostic criteria, including findings on plain radiograph, magnetic resonance imaging, and bone scans (Sugano et al. 1999). According to the classification system of the Japanese Investigation Committee of Health and Welfare, five hips in the ARO group and six in the THA group were classified as stage $3 \mathrm{~A}$, meaning a collapse of less than $3 \mathrm{~mm}$. Four hips in the ARO group and four hips in the THA group were stage $3 \mathrm{~B}$, indicating a collapse of $3 \mathrm{~mm}$ or more. One hip in the THA group was stage 4, indicating the presence of osteoarthritic change (Sugano et al. 2002). According to the location of the necrotic lesion, five hips in the ARO group and three hips in the THA group were classified as type $\mathrm{C} 1$, indicating that the necrotic lesion occupied more than two-thirds of the weight-bearing portion, but did not extend to the acetabular edge. Four hips in the ARO group and eight hips in the THA group were classified as type $\mathrm{C} 2$, indicating that the necrotic lesion extended to the acetabular edge (Sugano et al. 2002).

\section{Indications for surgeries}

In general, the indications for ARO were less than 60 years of age and stage $3 \mathrm{~A}$ or $3 \mathrm{~B}$ ONFH with onethird or more of intact area in the posterior region of the femoral head. ARO was performed for male and female patients who preferred hip joint preservation after both ARO and THA were explained to them. Twelve of 20 patients had indications for ARO in this study. Of these patients, ARO was performed for nine patients. The surgical procedure of ARO was performed according to the originally described method (Sugioka 1978). Two Kirschner wires were inserted into the femoral neck through the center of the femur, perpendicular to the plane of the femoral neck. After radiographic control, the first osteotomy was made in the plane of the Kirchner wires. The second osteotomy was made directing to the first osteotomy plane, just above the lesser trochanter. The proximal fragment was then rotated such that the new weight-bearing areas are well apposed. Two or three lag screws were then inserted to maintain fixation in this position (Figs. 1; 2). Patients usually began using wheelchairs 2 days after surgery. Partial weight-bearing walking was permitted approximately 5 weeks after surgery, followed by gradual increase in weight bearing. Full weight-bearing walking was basically permitted at around 6 months postoperatively. On the other hand, THA is indicated for patients who have no indication or do not wish to undergo ARO. Full weight-bearing walking was usually permitted a few days after surgery.

\section{Clinical assessment}

The preoperative assessment was performed at the time of hospital admission for surgery, and the postoperative assessment was performed at the time of final follow-up in the outpatient clinic.

The Japanese Orthopaedic Association (JOA) hip score evaluates pain $(0-40)$, gait $(0-20)$, activities of daily living $(0-20)$, and range of motion $(0-20)$. The possible range of values is $0-100$, with the higher values reflecting a better state (Takeda et al. 2006). 
Table 1 Demographic characteristics of the two groups

\begin{tabular}{|c|c|c|c|c|}
\hline Patient no. & Group & Age (years) & Sex & Etiology \\
\hline 1 & $\mathrm{ARO}$ & 38 & Male & Alcohol \\
\hline 2 & ARO & 41 & Male & Alcohol \\
\hline 3 & $\mathrm{ARO}$ & 45 & Male & Alcohol \\
\hline 4 & ARO & 36 & Male & Alcohol \\
\hline 5 & ARO & 28 & Male & Steroids \\
\hline 6 & ARO & 38 & Male & Idiopathic \\
\hline 7 & $\mathrm{ARO}$ & 32 & Male & Steroids \\
\hline 8 & ARO & 40 & Male & Steroids \\
\hline 9 & $\mathrm{ARO}$ & 40 & Female & eroids \\
\hline 10 & THA & 29 & Female & Steroids \\
\hline 11 & THA & 57 & Female & Steroids \\
\hline 12 & THA & 60 & Male & Alcohol \\
\hline 13 & THA & 32 & Female & Steroids \\
\hline 14 & THA & 47 & Female & Steroids \\
\hline 15 & THA & 55 & Male & Alcohol \\
\hline 16 & THA & 31 & Female & Idiopathic \\
\hline 17 & THA & 50 & Female & Alcohol \\
\hline 18 & THA & 30 & Female & Steroids \\
\hline 19 & THA & 58 & Female & Steroids \\
\hline 20 & THA & 41 & Female & Alcohol \\
\hline
\end{tabular}

$A R O$ transtrochanteric anterior rotational osteotomy, THA total hip arthroplasty
The Oxford hip score (OHS) is a disease-specific 12 -item questionnaire evaluating both pain (6 items) and physical function (6 items) of the hip joint in relation to daily activities (Dawson et al. 1996). The six items (OHS $1,6,8,10,11$, and 12) indicate pain severity, while the other six items (OHS 2, 3, 4, 5, 7, and 9) indicate physical function. The two items, OHS 5 and 7, are related to the bilateral hip function. This questionnaire was originally written in English, and Uesugi et al. translated the questionnaire into Japanese (Uesugi et al. 2009). Their translation was subsequently validated by a back translation as described in a previous report (Uesugi et al. 2009). The original version from 1996 was updated in 2007 introducing a new scoring system. The new scoring was supported by the original authors: item scores range from 0 to 4 (worst to best) with overall scores ranging from 0 to 48 , where a score of 48 is the best possible score (Nilsdotter and Bremander 2011). We also modified the classification of OHS described by Kalairajah et al. as follows: excellent, 42-48 points; good, 34-41 points; fair, 27-33 points; and poor, less than 27 points (Kalairajah et al. 2005).

The Medical Outcomes Study Short Form 36 (SF-36; SF-36v2 Standard, Japanese) was used to evaluate quality of life. It is composed of 36 items that measure general

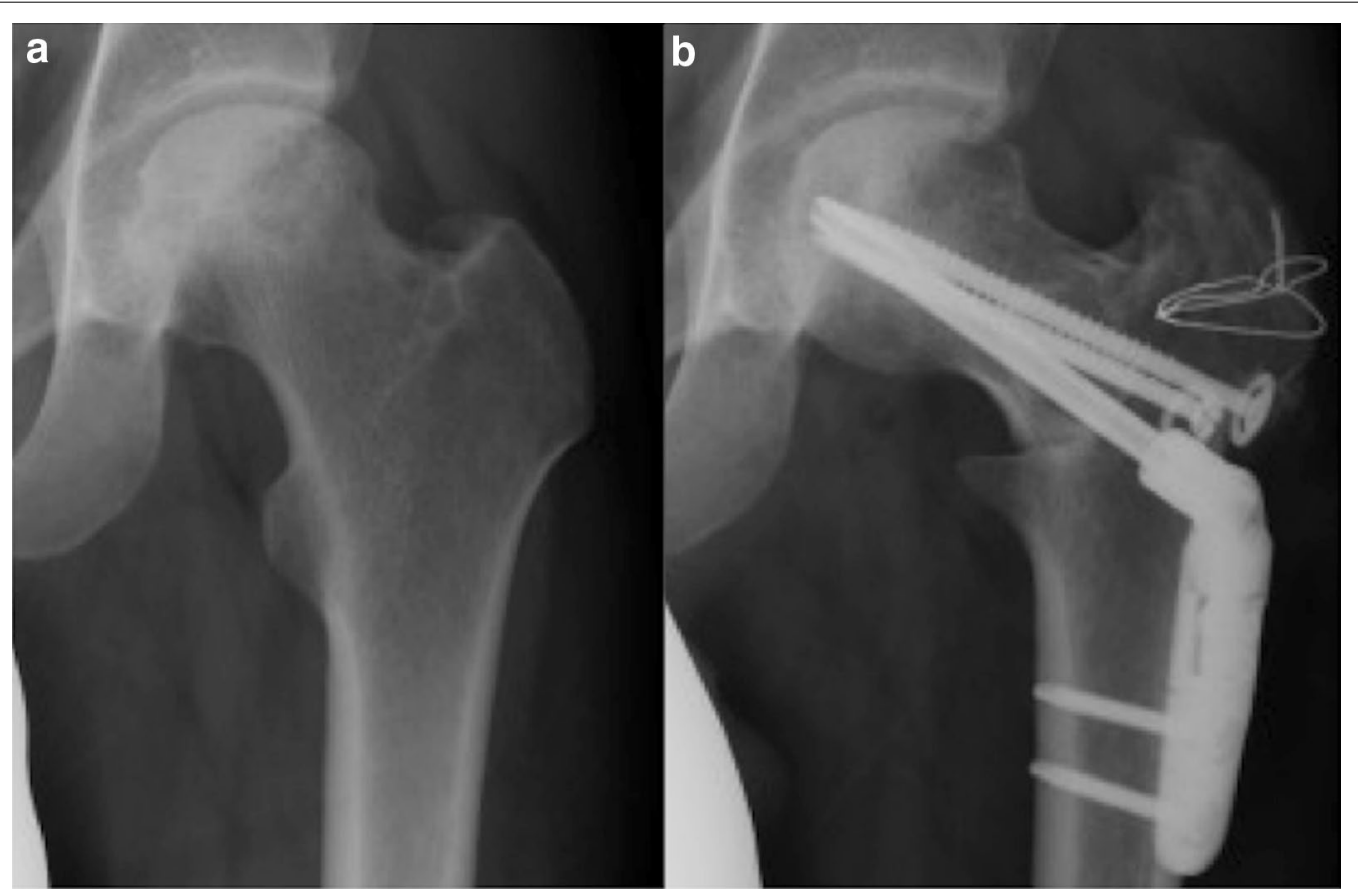

Fig. 1 A 28-year-old male with unilateral ONFH treated with ARO (Patient 5). a Preoperative anteroposterior radiograph shows left femoral head collapse and bone sclerosis, which indicates ONFH with stage $3 \mathrm{~A}$ and type C1. b Anteroposterior radiograph after ARO of the left hip 


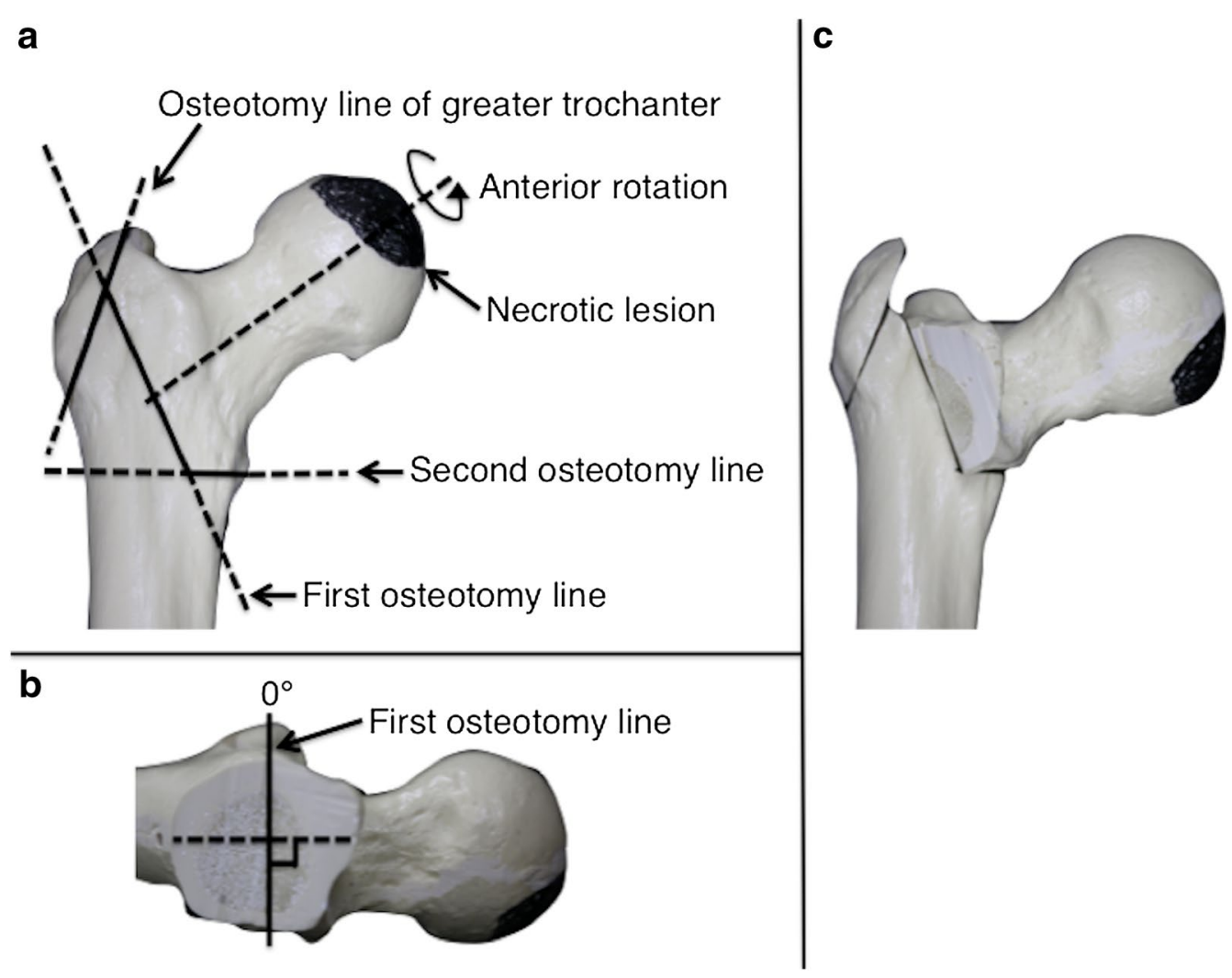

Fig. 2 Three-dimensional illustrations of the technique for ARO using a bone model. Anteroposterior $\mathbf{a}$ and lateral b views show the two osteotomy lines. The first osteotomy is made in the plane perpendicular to the femoral neck, and the second line is made directing to the first osteotomy plane, just above the lesser trochanter. Anteroposterior view c showing the bone model after $90^{\circ}$ of anterior rotation

health on eight subscales (Fukuhara et al. 1998). We derived a physical component summary (PCS) score and a mental component summary (MCS) score from the SF-36; then, an adjusted score was calculated by normbased scoring, which facilitates comparison of each score with that of the Japanese population norm.

\section{Statistical analysis}

The JOA score, OHS, and SF-36 were compared using Student's $t$ test. The distribution of ONFH etiology in the two groups was compared using the Chi square test. $P$ values less than 0.05 were considered significant. All statistical analyses were performed using the JMP Pro 11 software package (SAS Institute, Cary NC, USA).

\section{Results}

Regarding the etiology of ONFH, corticosteroid use was associated with four hips in the ARO group and six hips in the THA group. Alcohol abuse was associated with four hips in each group. One hip in each group did not have any associated factors $(\mathrm{P}=0.90)$ (=idiopathic ONFH).
The preoperative JOA score was $58.1 \pm 20.5$ and $48.6 \pm 16.7$ points in the ARO and THA groups, which significantly improved to $81.9 \pm 14.5$ and $86.6 \pm 10.6$ points at the final follow-up, respectively (Fig. 3). During the follow-up period, one patient in the ARO group presented separation of the greater trochanter 1 week postoperatively, resulting in osteosynthesis 4 weeks postoperatively. There were no other complications in both groups.

The preoperative OHS was $29.1 \pm 10.9$ points (excellent: 1 hip, good: 1 hip, fair: 4 hips, and poor: 3 hips) in the ARO group and $21.9 \pm 9.6$ points in the THA group (good: 3 hips, poor: 8 hips), which significantly improved to $38.4 \pm 9.4$ points (excellent: 5 hips, good: 3 hips, and poor: 1 hip) and $40.3 \pm 5.1$ points (excellent: 5 hips, good: 4 hips, and fair: 2 hips) at the final follow-up, respectively (Fig. 3). At the final follow-up, there were significant improvements in 'usual pain', 'getting in/out of a car', and 'ascending stairs' in the ARO group. Other items were also improved although not significantly. Notably, only the limp item was rated with a low score in this group. In the THA group, significant improvements were found 

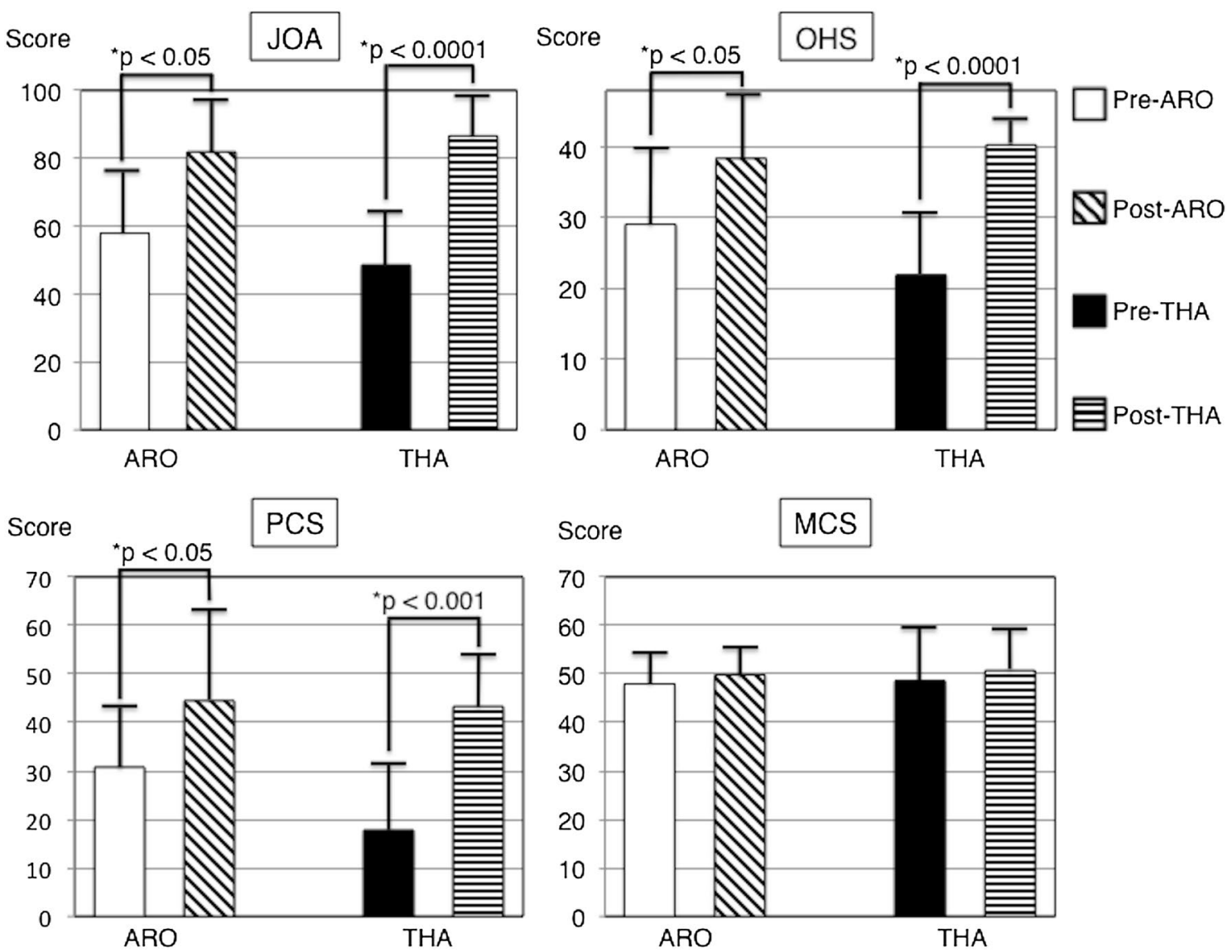

Fig. 3 Comparison of JOA score, OHS, PCS and MCS scores in the ARO and the THA groups. JOA Japanese Orthopaedic Association, OHS Oxford hip score, PCS physical component summary, MCS mental component summary, ARO transtrochanteric anterior rotational osteotomy, THA total hip arthroplasty

in all of the items, except for 'sudden pain. The 'sudden pain' item was also improved although not significantly (Fig. 4).

The preoperative PCS score was $30.8 \pm 12.8$ and $17.8 \pm 14.5$ points in the ARO and THA groups, which significantly improved to $44.5 \pm 10.6$ and $43.3 \pm 10.4$ points at the final follow-up, respectively. On the other hand, the preoperative MCS score was $48.0 \pm 8.5$ points in the ARO group and $48.6 \pm 11.3$ points in the THA group, both of which remained unchanged at the final follow-up (Fig. 3). Regarding each SF-36 subscale, there were significant improvements in two of the eight subscales ('role physical' and 'bodily pain') in the ARO group, while in the THA group, five of the eight subscales ('physical functioning' 'role physical' 'bodily pain' 'social functioning', and 'role emotional') were found to be significantly improved (Fig. 5). In comparison with the PCS score for the normal Japanese population, four hips (44.4\%) in the ARO group and four hips (36.4\%) in the THA group were within the Japanese norm (PCS score of
50). Regarding the MCS score for normal Japanese population, three hips (33.3\%) in the ARO group and five hips (45.5\%) in the THA group were within the Japanese norm (MCS score of 50).

\section{Discussion}

Reportedly, patients that undergo THA are generally satisfied with the outcomes (Wiklund and Romanus 1991; Learmonth et al. 2007), while the satisfaction level of patients that undergo ARO is relatively unknown. In the current prospective study, we found significant improvements in the hip joint functions according to patientreported outcomes not only in patients who underwent THA but also in those who underwent ARO during a short-term follow-up period. Although full weight-bearing walking is basically permitted at around 6 months after ARO, the results of the current study show that joint-preserving surgery needs to be considered for young patients in order to avoid or delay THA (Ikemura et al. 2009). 


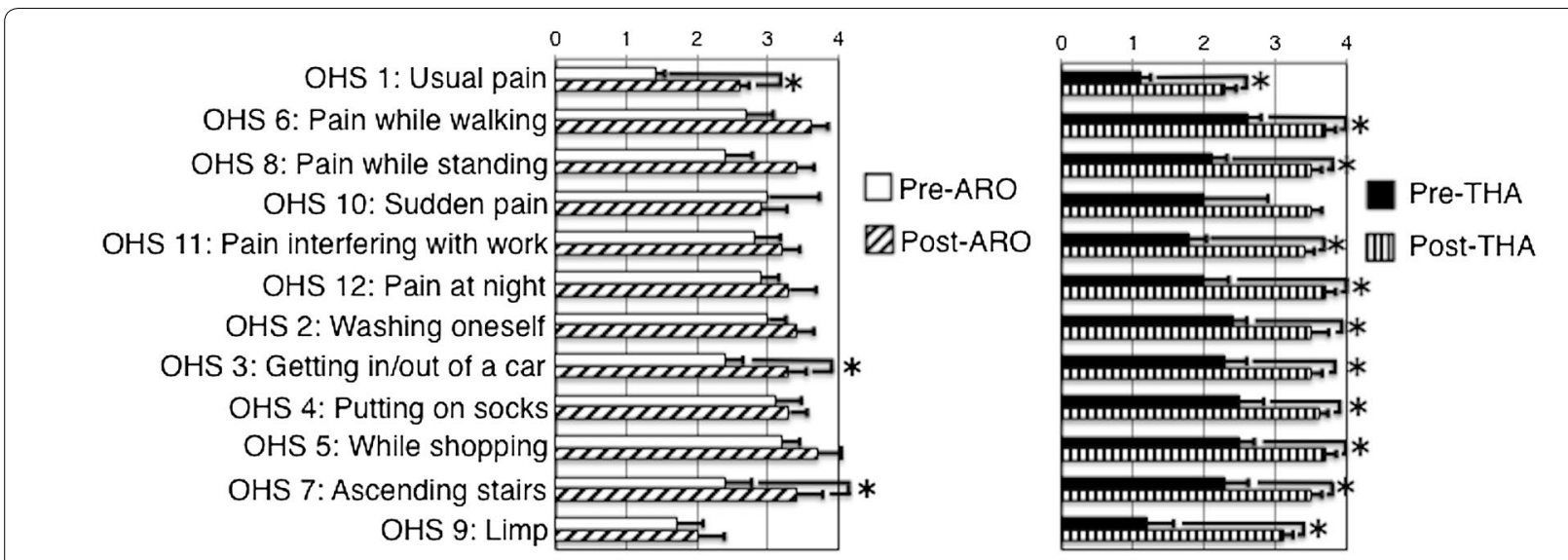

Fig. 4 Comparison of perioperative OHS subscales in the ARO and the THA groups. *P value $<0.05$. Error bars standard deviation. OHS Oxford hip score, $A R O$ transtrochanteric anterior rotational osteotomy, THA total hip arthroplasty

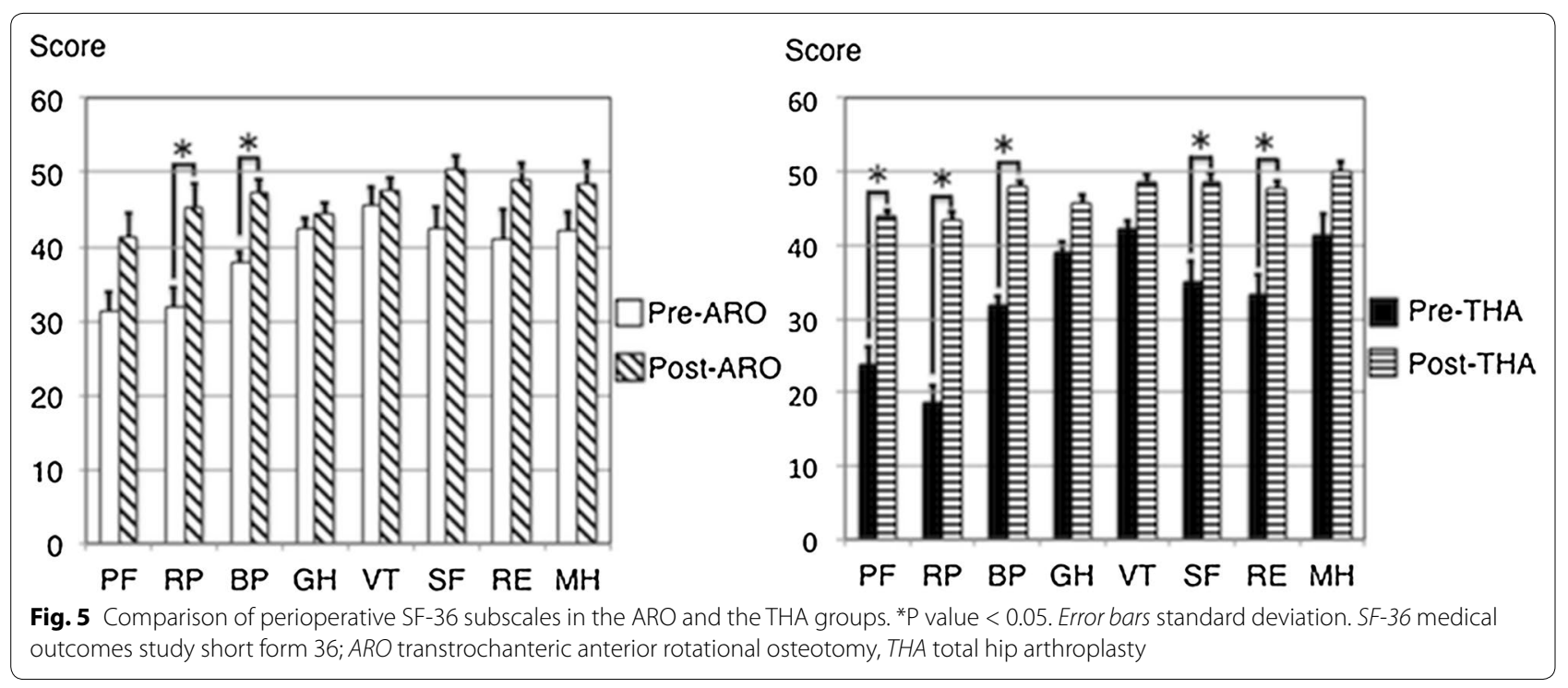

Several studies have reported patient-reported outcomes of ONFH treatment (Seki et al. 2008; Motomura et al. 2010; Kang et al. 2013). Seki et al. evaluated the SF-36 in 41 ONFH patients at a mean follow-up of 5.2 years after transtrochanteric rotational osteotomy (TRO) and in 19 patients at a mean follow-up of 4.1 years after THA. They reported that average PCS and MCS scores after TRO were 39.4 and 49.6 points, and average PCS and MCS scores after THA were 39.1 and 50.3 points, respectively (Seki et al. 2008). Kang et al. evaluated the SF-36 in 24 systemic lupus erythematosus patients with ONFH at a mean follow-up of 67.5 months after THA, reporting average PCS and MCS scores after THA of 42.2 and 49.5, respectively (Kang et al. 2013). In our study, the postoperative PCS score was 44.5 in the
ARO group and 43.3 in the THA group, both of which were slightly higher than those in the previous reports. These slight differences in outcomes may be explained by the fact that participants of the current study had symptomatic ONFH with asymptomatic contralateral hip. In our study, 22 (52.4\%) of 42 patients presented contralateral hip pain during the follow-up period, and thus were excluded from the present analysis. However, in the previous reports, ONFH patients with symptomatic contralateral hip were not strictly excluded, which might lead to lower PCS scores.

In the current study, of all OHS items assessed by patients in the ARO group at the final follow-up, the only item that did not reflect improvement was the 'limp' item. This finding indicates that limping was often experienced 
by patients that underwent ARO. In ARO surgery, a varus positioning of the femoral neck-shaft angle is frequently intended to acquire enough intact weight-bearing area of the femoral head, which can thereby cause shortening of the leg (Sugioka 1978; Dean and Cabanela 1993; Ha et al. 2010). We therefore consider that one of the major reasons for limping after ARO is the resulting leg length discrepancy caused by the intended varus neck-shaft-angle created during ARO. For this reason, surgeons should clearly explain to their patients that limping may present secondary to a leg length discrepancy after ARO.

The main limitation of this study was the small sample size. However, we only included ONFH patients with asymptomatic contralateral hip during the follow-up. We therefore believe that the current results reflect the real outcomes of the surgery performed without any influence of the contralateral hip symptoms. A future multicenter study should be conducted with a similar protocol to validate our results. Second, the postoperative follow-up period was short in this study (range: 1-6 years). However, the postoperative complication in both groups was only one instance of greater trochanter separation. Therefore, we believe that these differences have little effect on the result of this study. Third, we evaluated the functional status only at the time of hospital admission for surgery and final follow-up. Considering that full weight-bearing walking was basically permitted at around 6 months after ARO, at least 1 year seems necessary to evaluate postoperative functional outcomes.

\section{Conclusions}

The short-term patient-reported outcomes of this study suggested that both ARO and THA for ONFH resulted in significantly improved postoperative hip joint function.

\section{Abbreviations \\ THA: total hip arthroplasty; ONFH: osteonecrosis of the femoral head; ARO: transtrochanteric anterior rotational osteotomy; OHS: Oxford hip score; SF-36: short form 36; BMI: body mass index; JOA: Japanese Orthopaedic Association; PCS: physical component summary; MCS: mental component summary; TRO: transtrochanteric rotational osteotomy.}

\section{Authors' contributions}

YK and GM performed literature review and drafted the manuscript. TY and YI supervised the writing. KK and KS were contributors in writing the manuscript. All authors read and approved the final manuscript.

\section{Author details}

${ }^{1}$ Department of Orthopaedic Surgery, Graduate School of Medical Sciences, Kyushu University, 3-1-1 Maidashi, Higashi-ku, Fukuoka 812-8582, Japan.

2 Department of Orthopaedic Surgery, Faculty of Medicine, Fukuoka University, 7-45-1 Nanakuma, Jonan-ku, Fukuoka, Japan. ${ }^{3}$ Department of Orthopaedic Surgery, Kyushu Rosai Hospital, 1-1 Sonekita-machi, Kokuraminami-ku, Kitakyushu 800-0296, Japan.

\section{Acknowledgements}

This work was partially supported by the Practical Research Project for Rare/Intractable Diseases from Japan Agency for Medical Research and
Development (AMED) and a grant-in-aid in Scientific Research (Grant 16K10906) from the Japan Society for the Promotion of Science.

\section{Competing interests}

The authors declare that they have no competing interests.

\section{Ethical approval}

The present prospective study was approved by our institutional review board (25-265) and was performed in accordance with the ethical standards as laid down in the 1964 Helsinki Declaration and its later amendments or comparable ethical standards.

\section{Informed consent}

All patients in this study were informed that both data and analysis would be submitted for publication and gave their consent.

Received: 2 March 2016 Accepted: 18 October 2016

Published online: 26 October 2016

\section{References}

Dawson J, Fitzpatrick R, Carr A, Murray D (1996) Questionnaire on the perceptions of patients about total hip replacement. J Bone Joint Surg Br 78:185-190

Dean MT, Cabanela ME (1993) Transtrochanteric anterior rotational osteotomy for avascular necrosis of the femoral head. Long-term results. J Bone Joint Surg Br 75:597-601

Fukuhara S, Bito S, Green J, Hsiao A, Kurokawa K (1998) Translation, adaptation, and validation of the SF-36 health survey for use in Japan. J Clin Epidemiol 51:1037-1044

Fukushima W, Fujioka M, Kubo T, Tamakoshi A, Nagai M, Hirota Y (2010) Nationwide epidemiologic survey of idiopathic osteonecrosis of the femoral head. Clin Orthop 468:2715-2724

Ha YC, Kim HJ, Kim SY, Kim KC, Lee YK, Koo KH (2010) Effects of age and body mass index on the results of transtrochanteric rotational osteotomy for femoral head osteonecrosis. J Bone Joint Surg Am 92:314-321

Ikemura S, Yamamoto T, Nakashima Y, Mawatari T, Motomura G, Iwamoto Y (2009) Transtrochanteric anterior rotational osteotomy for osteonecrosis of the femoral head in patients 20 years or younger. J Pediatr Orthop 29:219-223

Inao S, Ando M, Gotoh E, Matsuno T (1999) Minimum 10-year results of Sugioka's osteotomy for femoral head osteonecrosis. Clin Orthop Relat Res 368:141-148

Kalairajah Y, Azurza K, Hulme C, Molloy S, Drabu KJ (2005) Health outcome measures in the evaluation of total hip arthroplasties - a comparison between the Harris hip score and the Oxford hip score. J Arthroplasty 20:1037-1041

Kang Y, Zhang ZJ, Zhao XY, Zhang ZQ, Sheng PY, Liao WM (2013) Total hip arthroplasty for vascular necrosis of the femoral head in patients with systemic lupus erythematosus: a midterm follow-up study of 28 hips in 24 patients. Eur J Orthop Surg Traumatol 23:73-79

Learmonth ID, Young C, Rorabeck C (2007) The operation of the century: total hip replacement. Lancet 370:1508-1519

Mankin HJ (1992) Nontraumatic necrosis of bone (osteonecrosis). N Engl J Med 326:1473-1479

Mont MA, Hungerford DS, Maryland B (1995) Non-traumatic osteonecrosis of the femoral head: ten years later. J Bone Joint Surg Am 77:459-474

Mont MA, Jones LC, Hungerford DS (2006) Nontraumatic avascular necrosis of the femoral head. J Bone Joint Surg Am 88:1117-1132

Motomura G, Yamamoto T, Suenaga K, Nakashima Y, Mawatari T, Ikemura S, Iwamoto Y (2010) Long-term outcome of transtrochanteric anterior rotational osteotomy for osteonecrosis of the femoral head in patients with systemic lupus erythematosus. Lupus 19:860-865

Nilsdotter A, Bremander A (2011) Measures of hip function and symptoms: Harris hip score (HHS), hip disability and osteoarthritis outcome score (HOOS), Oxford hip score (OHS), Lequesne index of severity for osteoarthritis of the hip (LISOH), and American Academy of Orthopedic Surgeons (AAOS), hip and knee questionnaire. Arthritis Care Res (Hoboken) 63(11):200-207 
Seki T, Hasegawa Y, Masui T, Yamaguchi J, Kanoh T, Ishiguro N, Kawabe K (2008) Quality of life following femoral osteotomy and total hip arthroplasty for nontraumatic osteonecrosis of the femoral head. J Orthop Sci 13:116-121 Sugano N, Kubo T, Takaoka K, Ohzono K, Hotokebuchi T, Matsumoto T, Igarashi H, Ninomiya S (1999) Diagnostic criteria for non-traumatic osteonecrosis of the femoral head. A multicentre study. J Bone Joint Surg Br 81:590-595 Sugano N, Atsumi T, Ohzono K, Kubo T, Hotokebuchi T, Takaoka K (2002) The 2001 revised criteria for diagnosis, classification, and staging of idiopathic osteonecrosis of the femoral head. J Orthop Sci 7:601-605

Sugioka Y (1978) Transtrochanteric anterior rotational osteotomy of the femoral head in the treatment of osteonecrosis affecting the hip: a new osteotomy operation. Clin Orthop Relat Res 130:191-201
Takeda H, Kamogawa J, Sakayama K, Kamada K, Tanaka S, Yamamoto H (2006) Evaluation of clinical prognosis and activities of daily living using functional independence measure in patients with hip fractures. J Orthop Sci 11:584-591

Uesugi Y, Makimoto K, Fujita K, Nishii T, Sakai T, Sugano N (2009) Validity and responsiveness of the Oxford hip score in a prospective study with Japanese total hip arthroplasty patients. J Orthop Sci 14:35-39

Wiklund I, Romanus B (1991) A comparison of quality of life before and after arthroplasty in patients who had arthrosis of the hip joint. J Bone Joint Surg Am 73:765-769

\section{Submit your manuscript to a SpringerOpen ${ }^{\circ}$ journal and benefit from:}

- Convenient online submission

- Rigorous peer review

- Immediate publication on acceptance

- Open access: articles freely available online

- High visibility within the field

- Retaining the copyright to your article 